\title{
AN ANNULAR FUNCTION WHICH IS THE SUM OF TWO NORMAL FUNCTIONS
}

\author{
PETER LAPPAN
}

\begin{abstract}
It is known that a nonconstant normal function cannot have a Koebe value. An example is presented of an annular function which is the sum of two normal holomorphic functions. This example shows that a sum of two normal functions can result in a nonconstant function which has the Koebe value $\infty$.
\end{abstract}

1. Let $D$ denote the unit disc and $C$ the unit circle in the complex plane. A function $f$ meromorphic in $D$ is said to be a normal function if the family of functions $\{f(S(z)): S \in \mathscr{S}\}$ is a normal family, where $\mathscr{S}$ is the collection of all conformal mappings of $D$ onto itself. Let $N$ denote the set of positive integers and let $Z$ denote the set of all integers. A function $f$ which is meromorphic in $D$ is said to have the Koebe value $\alpha$ if there exists a sequence of Jordan $\operatorname{arcs}\left\{J_{n}\right\}$ in $D$ such that

(i) there exist distinct radii $R_{1}$ and $R_{2}$ of $D$ such that, for each $n \in N$ the $\operatorname{arc} J_{n}$ meets both $R_{1}$ and $R_{2}$;

(ii) if $m_{n}=\inf \left\{|z|: z \in J_{n}\right\}$, then $m_{n} \rightarrow 1$ as $n \rightarrow \infty$; and

(iii) if $\alpha$ is finite and if $u_{n}=\sup \left\{|f(z)-\alpha|: z \in J_{n}\right\}$ then $u_{n} \rightarrow 0$ as $n \rightarrow \infty$; while if $\alpha=\infty$ and if $v_{n}=\inf \left\{|f(z)|: z \in J_{n}\right\}$, then $v_{n} \rightarrow \infty$ as $n \rightarrow \infty$.

A function $f$ holomorphic in $D$ is called an annular function if there exists a sequence of Jordan curves $\left\{J_{n}\right\}$ in $D$ such that for each $n \in N$ the curve $J_{n}$ is in the interior of $J_{n+1}$ and conditions (ii) and (iii) are satisfied with $\alpha=\infty$.

It has been proved by Bagemihl and Seidel $[1$, Theorem 1, p. 10] that a nonconstant normal meromorphic function cannot have a Koebe value, either finite or infinite. The author [2, Theorem 5, p. 191] has shown that a sum of two normal functions need not result in a normal function. Further, since the Nevanlinna characteristic function $T(r)$ for a normal function satisfies $T(r)=O(\log (1 /(1-r))$, the sum of two normal holomorphic functions must result in a function which is either a constant or is in Mac Lane's class A (see [4, pp. 43-44]), and such a function cannot have a finite Koebe value. We prove here that a sum of two normal homomorphic functions actually can have $\infty$ as a Koebe value in the strongest possible sense.

Received by the editors February 5, 1973 and, in revised form, September 17, 1973. AMS (MOS) subject classifications (1970). Primary 30A74, 30A58; Secondary 30A72, 30A76.

Key words and phrases. Normal function, annular function, Koebe value, harmonic measure.

(c) American Mathematical Society 1974 
2. If $\Gamma$ is a crosscut of $D$ with $0 \notin \Gamma$ and if $w$ is a point of $C$ we say that $\Gamma$ separates 0 from $w$ if the component of $D-\Gamma$ which contains 0 does not have $w$ as a boundary point. The following lemma is basic.

Lemma. Let $h$ be a function holomorphic in $D$. If for each point $w \in C$ and each $n \in N$ there exists a crosscut $\Lambda(w, n)$ such that $|h(z)| \geqq n$ for each $z \in \wedge(w, n)$ and $\bigwedge(w, n)$ separates 0 from $w$, then $h$ is an annular function.

Proof. For each $n \in N$ let $D_{n}$ be the component of $\{z \in D:|h(z)|<n\}$ containing the origin. (It is possible that $D_{n}=\varnothing$ for a finite number of $n$ 's.) Let $w$ be any point of $C$. For each $n \in N, \wedge(w, n) \cap D_{n}=\varnothing$ so that, if $K_{n}$ denotes the boundary of $D_{n}$ we have that $w \notin K_{n}$. Let $n_{0}$ be the least positive integer $n$ for which $D_{n} \neq \varnothing$ and set $J_{n}=K_{n_{0}}$ for $n \leqq n_{0}$ and $J_{n}=K_{n}$ for $n>n_{0}$. Then $J_{n}$ is a Jordan curve, $|h(z)| \geqq n$ for $z \in J_{n}$, and $C \cap J_{n}=\varnothing$. Thus $h$ is an annular function.

THEOREM. There exist two normal holomorphic functions $f$ and $g$ in $D$ such that the sum $h=f+g$ is an annular function. Further, $g$ can be chosen to be a function with positive real part.

The proof of this theorem involves many detailed considerations, but the idea is basically to choose two normal holomorphic functions $f$ and $g$ such that the sum $h$ satisfies the hypotheses of the Lemma. We note that, by renumbering the crosscuts $\Lambda(w, n)$, if necessary, we can replace the condition that $|h(z)| \geqq n$ on $\wedge(w, n)$ by the more general condition that for each fixed $w \in C$ we have

$$
\lim _{n \rightarrow \infty}(\inf \{|h(z)|: z \in \Lambda(w, n)\})=\infty .
$$

Let $M(z)$ denote the elliptic modular function in $D$. Since $M(z)$ omits the values 0,1 , and $\infty$ in $D$, we can take $\log M(z)$ and $\log \log M(z)$ to be holomorphic in $D$ by assigning an appropriate value to the origin. Further, since both $\log M(z)$ and $\log \log M(z)$ omit a countable set of values, both are normal functions. We shall be concerned with the real part of the $\operatorname{logarithm}$ function, and we use the notation $\operatorname{Re}(\log z)=\ln |z|$. Let $A_{1}$, $A_{2}, A_{3}$, and $A_{4}$ be the subsets of $C$ defined by

$A_{1}=\{w \in C: M(z)$ has an asymptotic value either 0 or $\infty$ at $w\}$, $A_{2}=\{w \in C: \log M(z)$ has the asymptotic value 0 at $w\}$, $A_{3}=\{w \in C$ : for each $n \in N$ there exists a crosscut $\wedge(w, n)$ of $D$ which separates 0 from $w$ and such that $\ln |\log M(z)|>\ln n \pi$ for $z \in \wedge(w, n)\}$,

and

$$
A_{4}=C-\left(A_{1} \cup A_{2} \cup A_{3}\right) \text {. }
$$


We may assume, without loss of generality, that $1 \in A_{2}$. We note that both $A_{1}$ and $A_{2}$ are countable sets. Since the components of the set of $z$ defined by $\operatorname{Arg} M(z)=k \pi, k \in N$, are all crosscuts of $D$ which are arcs of circles orthogonal to $C$, and since the radial projection of these components onto $C$ consists of all but a subset of $C$ of measure zero, it follows that $A_{3}$ has measure $2 \pi$ so that $A_{4}$ has measure zero (see [3, p. 123] for a more detailed account of this situation).

We claim that $A_{1} \subset A_{3}$. If $w \in A_{1}$, then for each $n \in Z$ there exists a crosscut $\alpha(w, n)$ of $D$ such that $\operatorname{Arg} M(z)=n \pi$ for $z \in \alpha(w, n)$ and $\alpha(w, n)$ is an arc of a circle orthogonal to $C$ at $w$. For each $n \in N$, let $G_{n}$ be the component of $D-(\alpha(w, n) \cup \alpha(w,-n))$ having both $\alpha(w, n)$ and $\alpha(w,-n)$ as boundary arcs of $G_{n}$. Then $|\ln | M(z)|| \rightarrow \infty$ as $z \rightarrow w$ from within $G_{n}$. Thus, by taking an appropriate crosscut of $G_{n}$, and combining this crosscut with appropriate subarcs of $\alpha(w, n)$ and $\alpha(w,-n)$ in the obvious manner, we obtain a crosscut $\wedge(w, n)$ of $D$ which separates 0 from $w$ and is such that $\ln |\log M(z)|>\ln n \pi$ for $z \in \Lambda(w, n)$. Since the process can be done for each $n \in N$, we have that $w \in A_{3}$.

Now, for each $n \in N$, let $E_{n}$ be the component of $\{z \in D: \ln |\log M(z)|<$ $\ln n \pi\}$ which contains the origin. (It is possible that $E_{n}=\varnothing$ for a finite number of $n$.) If $B_{n}$ is the collection of all points of $C$ which are accessible boundary points of $E_{n}$, then $B_{n}$ is a closed subset of $C$ (see [3, Lemma, p. 121]). Clearly, for each $n \in N, B_{n} \cap A_{3}=\varnothing$. It is possible that $A_{2} \cap$ $B_{n} \neq \varnothing$ for some $n$, but we claim that the set $B_{n}^{\prime}=B_{n}-A_{2}$ is a closed set for each $n \in N$. For if $w \in A_{2}$, then for each $n \in Z$ there exists a crosscut $\mathscr{B}(w, n)$ of $D$ such that $\mathscr{B}(w, n)$ has one endpoint at $w, \mathscr{B}(w, n)$ is an arc of a circle orthogonal to $C$ at $w$, and $\operatorname{Arg} \log M(z)=n \pi$ for $z \in \mathscr{B}(w, n)$. Thus, for each $n \in N$ and $z \in \mathscr{B}(w, n) \cup \mathscr{B}(w,-n)$ we have the $\ln |\log M(z)|>$ $\ln n \pi$ so that if $w \in B_{n} \cap A_{2}$, then $w$ is an isolated point of $B_{n}$. It follows that $B_{n}^{\prime}$ is a closed set for each $n \in N$. It should be further noted that for $w \in A_{2}, n \in Z$, the endpoint of $\mathscr{B}(w, n)$ other than $w$ is a point of $A_{1}$, and hence is not a point of $B_{|n|}^{\prime}$.

If $w \in A_{\mathbf{2}}, n \in N$, and if $H_{n}$ is the component of $D-(\mathscr{B}(w, n) \cup \mathscr{B}(w,-n))$ having both $\mathscr{B}(w, n)$ and $\mathscr{B}(w,-n)$ as boundary arcs, then

$$
\ln |\log M(z)| \rightarrow-\infty
$$

as $z \rightarrow w$ from within $H_{n}$. By taking an appropriate crosscut of $H_{n}$ and combining this with appropriate subarcs of $\mathscr{B}(w, n)$ and $\mathscr{B}\left(w^{\prime},-n\right)$ in the obvious manner, we obtain a crosscut $\Lambda(w, n)$ of $D$ which separates 0 from $w$ and which has both its endpoints in $A_{1}$ such that $|\log \log M(z)|>$ $\ln n \pi$ for $z \in \wedge(w, n)$.

Since $A_{2}$ is a countable set, let $A_{2}=\left\{w_{1}, w_{2}, w_{3}, \cdots, w_{n}, \cdots\right\}$ and 
let

$$
P_{n}=\bigcup_{j=1}^{n}\left(\bigcup_{k=1}^{\infty} \wedge\left(w_{j}, k\right)\right) \cup\left\{w_{1}, w_{2}, w_{3}, \cdots, w_{n}\right\}
$$

and let

$$
Q_{n}=P_{n} \cup\{z \in D:|z| \leqq 1-1 / n\} .
$$

Then $Q_{n}$ is a compact subset in the closure of $D, B_{n}^{\prime}$ is a compact subset of $C$, and $Q_{n} \cap B_{n}^{\prime}=\varnothing$. Let $\delta_{n}$ denote the distance between $Q_{n}$ and $B_{n}^{\prime}$, let $\delta_{n}(\theta)$ denote the distance from $e^{i \theta}$ to $Q_{n}$, and let $I_{n}=\left\{\theta \in(0,2 \pi): \delta_{n}(\theta)>\right.$ $\left.\delta_{n} / 2\right\}$. We note that

$$
\int_{I_{n}} d \theta / \delta_{n}(\theta)<\infty
$$

We will make repeated use of the following general principle. If $Q$ is a compact subset in the closure of $D$ and if $A$ is an arc of $C$ where

$$
A=\left\{e^{i \theta}: \theta_{1}<\theta<\theta_{2}\right\}
$$

such that $Q \cap A=\varnothing$, then for $z \in Q$ we have

$$
\log \frac{e^{i \theta_{1}}-z}{e^{i \theta_{2}}-z}=\int_{\theta_{2}}^{\theta_{1}} \frac{i e^{i \theta}}{e^{i \theta}-z} d \theta
$$

where the logarithm is taken with imaginary part between 0 and $2 \pi$ for $z \in Q$. From (2), it follows that

$$
\left|\log \frac{e^{i \theta_{1}}-z}{e^{i \theta_{2}}-z}\right| \leqq \int_{\theta_{1}}^{\theta_{2}} \frac{d \theta}{\left|e^{i \theta}-z\right|}=\int_{\theta_{1}}^{\theta_{2}} d \theta / \delta(\theta),
$$

where $\delta(\theta)$ denotes the distance from $e^{i \theta}$ to $Q$.

Returning to the sets $Q_{n}$ and $B_{n}^{\prime}, n \in N$, and setting $S_{n}=\{\theta \in(0,2 \pi)$ : $\left.e^{i \theta} \in B_{n}^{\prime}\right\}$-recall that $w=1 \in A_{2}$ and hence is not in $B_{n}^{\prime}$-then $S_{n} \subset I_{n}$ and $S_{n}$ is a compact set with measure zero since $B_{n}^{\prime} \subset A_{4}$. Hence, for each $n \in N$ there exist a finite number of disjoint open intervals $(a(n, j), b(n, j))$, $1 \leqq j \leqq m_{n}$, such that

$$
\begin{gathered}
S_{n} \subset \bigcup_{j=1}^{m}(a(n, j), b(n, j)) \subset I_{n}, \\
\sum_{j=1}^{m_{n}} \int_{a(n, j)}^{b(n, j)} d \theta / \delta_{n}(\theta)<(1 / 2)^{n+1} \\
\sum_{j=1}^{m_{n}}(b(n, j)-a(n, j))<(1 / 2)^{n+1},
\end{gathered}
$$

and such that none of the points $e^{i a(n, j)}$ or $e^{i b(n, j)}$ lies in $A_{2}$. By (3) and (4) 
we have that, for $n \in N, z \in Q_{n}$,

$$
\sum_{j=1}^{m_{n}}\left|\log \frac{e^{i a(n, j)}-z}{e^{i b(n, j)}-z}\right|<(1 / 2)^{n+1} .
$$

Now let

$$
g_{n, j}(z)=(i / \pi) \log \frac{e^{i a(n, j)}-z}{e^{i b(n, j)}-z}+(1 / 2)(b(n, j)-a(n, j)) .
$$

Then $g_{n, j}(z)$ has the property that its real part is the harmonic measure of the arc $T_{n, j}=\left\{e^{i \theta}: a(n, j)<\theta<b(n, j)\right\}$. It follows that $\operatorname{Re} g_{n, j}(z)>0$ for all $z \in D$, and $\operatorname{Re} g_{n, j}(z) \rightarrow 1$ as $z$ approaches any point of $T_{n, j}$ from within $D$. We define

$$
g(z)=\sum_{n=1}^{\infty} \sum_{j=1}^{m_{n}} g_{n, j}(z)
$$

By (5), (6), and the fact that $D \subset \cup Q_{n}$, we have that $g(z)$ is a function holomorphic in $D$, and $g(z)$ has positive real part in $D$ with $\operatorname{Re} g(z) \rightarrow+\infty$ as $z$ approaches any point of $A_{4}$, since any point of $A_{4}$ is in all but a finite number of the sets $B_{n}^{\prime}$. Further, for $z \in Q_{n}$, by (5), (6), and (7) we have that

$$
|g(z)| \leqq 1+\sup _{z \in Q_{n}} \sum_{k=1}^{n-1} \sum_{j=1}^{m_{n}}\left|g_{k, j}(z)\right|
$$

where the right side is a finite sum and is thus a fixed number for each fixed $n$. It follows that if $w \in A_{2}$, then $w=w_{q}$ for some natural number $q$ so that $w=w_{q} \in Q_{n}$ for $n \geqq q$ and this yields that $|\log \log M(z)+g(z)| \rightarrow \infty$ as $z \rightarrow w$ along $\bigcup_{k=1}^{\infty} \wedge(w, k)$. Since $g(z)$ has positive real part, for $z \in A_{3}$ we have that $|\log \log M(z)+g(z)| \rightarrow \infty$ as $z \rightarrow w$ along $\bigcup_{k=1}^{\infty} \wedge(w, k)$. It remains to obtain the same relationship for $w^{\prime} \in A_{4}$. However, we note that if $X=D-\left\{z \in D:|\log M(z)|<\frac{1}{2}\right\}$, then each point of $A_{4}$ is an accessible boundary point of $X$, and further, for each point $w^{\prime} \in A_{4}$ and each $n \in N$ there exists a crosscut $\bigwedge(w, n)$ of $D$ such that $\Lambda(w, n) \subset X \cap\{z \in D$ : $|z-w|<1 / n\}$. (The region $X$ is simply $D$ with some nearly circular regions deleted, where each of the regions deleted has only one point of contact with $C$ and each such point of contact is a point of $A_{2}$.) Each such $\wedge(w, n)$ separates 0 from $w$. But $\ln |\log M(z)|>\ln \left(\frac{1}{2}\right)$ for $z \in X$, and hence, since $\operatorname{Re} g(z) \rightarrow+\infty$ as $z \rightarrow w$ we have that $|\log \log M(z)+g(z)| \rightarrow \infty$ as $z \rightarrow w$ along $\bigcup_{k=1}^{\infty} \wedge(w, k)$. Thus (1) is established for each $w \in C$ and, by the second sentence following the statement of the Theorem, together with the Lemma, the Theorem is proved with $f(z)=\log \log M(z)$ and $g(z)$ as defined in (7).

We remark that, since $g$ is a function with positive real part it is a function of bounded type, so that the main burden of making $h$ an annular 
function falls on $f$. For if $\left\{J_{n}\right\}$ is the sequence of Jordan curves for $h$ satisfying (i), (ii), and (iii) for $\alpha=\infty$, and if $Y_{n}$ denotes the region bounded by $J_{n}$ for each $n$, then it is easy to show that if $\left\{\lambda_{n}\right\}$ is any sequence of real numbers tending to $\infty$, then the harmonic measure of the set $\left\{z \in J_{n}\right.$ : $\left.|g(z)| \geqq \lambda_{n}\right\}$ relative to $Y_{n}$ tends to zero as $n$ tends to $\infty$. Thus, the harmonic measure of $\left\{z \in J_{n}:|f(z)| \geqq \lambda_{n}\right\}$ relative to $Y_{n}$ tends to $2 \pi$ as $n \rightarrow \infty$ for some choice of $\lambda_{n}$. Thus $f$ is "almost" an annular function.

\section{REFERENCES}

1. F. Bagemihl and W. Seidel, Koebe arcs and Fatou points of normal functions, Comment. Math. Helv. 36 (1961), 9-18. MR 25 \#5183.

2. P. Lappan, Non-normal sums and products of unbounded normal functions, Michigan Math. J. 8 (1961), 187-192. MR 24 \#A1403.

3. - Some results on a class of holomorphic functions, Comm. Math. Univ. St. Paul 18 (1970), 119-124. MR 44 \#1810.

4. G. R. Mac Lane, Asymptotic values of holomorphic functions, Rice Univ. Studies 49 (1963), no. 1, 83 pp. MR 26 \#6419.

Department of Mathematics, Michigan State University, East lansing, MICHIGAN 48824 